\title{
ORTODOXIAS, DISIDENCIAS Y NUEVAS IDENTIDADES EN EL JUDAÍSMO ARGENTINO Y CHILENO'
}

\author{
Isaac Caro
}

Universidad Alberto Hurtado / Depto. de Ciencia Política y Relaciones Internacionales Universidad Arturo Prat / Instituto de Estudios Internacionales

Isaac.caro@gmail.com

\begin{abstract}
RESUMEN
En este artículo analizamos identidades y procesos que caracterizan al mundo judio contemporáneo en Argentina y Chile a partir de cuatro factores primordiales, que corren en paralelo y están interrelacionados: a) la constitución de identidades judías laicas, con elementos progresistas y liberales, sin que la religión sea necesariamente un elemento central de identificación; b) una tendencia a la consolidación de un espacio religioso ortodoxo, que puede alcanzar a un $10 \%$ de la población; c) la formación de nuevas identidades, muchas de ellas lejos de los marcos comunitarios institucionalizados; d) la consolidación de un debate que incluye tanto al judaísmo institucionalizado como al informal, que se plantea en torno a una crítica al modelo comunitario centralizado.
\end{abstract}

Palabras Clave: judaísmo, identidades, Argentina, Chile.

\begin{abstract}
In this paper, we consider different perspectives on Chilean and Argentinean Judaism, related to four main traits: a) the organization of Jewish and secular identities, with progressive and liberal components, although religion is not the central identity element; b) the consolidation of a religious orthodox space; c) the formation of new Jewish identities, that are not integrated into the structures of institutional Jewish communities; e) the emergence of some dissidence in Chilean and Argentinean Judaism, as part and parcel of a reaction to the official discourse about the Arab-Israeli Conflict.
\end{abstract}

KEY WoRDs: Judaism, Identities, Argentina, Chile.

Este articulo forma parte del Proyecto FONDECYT No 1050053, titulado "Movimientos, comunidades e identidades judias e islámicas en América Latina: los casos de Argentina, Brasil y Chile". Agradecimientos a Tomás Cabrera, Ingrid Hecker, Débora Roitman y Valeria Navarro. 


\section{INTRODUCCIÓN}

Uno de los problemas más difíciles de determinar en lo que se refiere a las identidades judías, y que explica la diversidad que caracteriza a las mismas, está relacionado con la propia definición de lo que se entiende por "judio". La cuestión de "quién es judio", está incorporada en la "Ley del Retorno", aprobada por el parlamento israelí en 1950, que lleva la firma del entonces primer ministro israelí, David Ben Gurión, donde se establece que es judio "todo aquel nacido de madre judía o convertido al judaísmo y no es miembro de otra religión" (Organización Sionista Mundial).

Los sectores más liberales buscan consolidar una concepción más amplia en la definición de quién es judio. De este modo, en diciembre de 2006, el parlamentario y líder del partido izquierdista Meretz, Yossi Beilin, presentó una propuesta, ante un comité ministerial, para que el Estado reconociera de manera oficial como judíos a las personas cuyo padre es de origen judío. De ser aprobada, esta propuesta pasaria a la Kneseet para su aprobación final. Se busca que el Ministerio del Interior considere en los documentos oficiales como judíos a las personas nacidas tanto de padre como de madre judía (La Palabra Israelita 2006, 12).

Existe también una tendencia que algunos judíos que se sienten más alejados de la tradición tomen posiciones distintas para autodefinir su judaísmo, sin que la religión tenga un lugar importante de identificación: "judaismo a tu manera", "orgullo de ser judio", "judios gay", "chilenos judios", versiones humorísticas del ser judío. En este sentido, se trata de un judaísmo pluralista, que no solo incluye la tradición religiosa -como defienden los sectores ortodoxos-, sino que incorpora diferentes versiones, agnósticas, seculares y ateas, que lo transforman en una tradición cultural y en una civilización (Sorj 2006:29) y que en algunos casos considera como más importante una identidad que puede ser humanista, planetaria, nacional o sexual.

Por el contrario, los partidos ortodoxos están luchando por restringir la definición de judio según la Ley del Retorno aun más, de modo de cambiar la palabra "convertido" por "convertido de acuerdo con la halajá" (ley religiosa judía). Esto debido a que la definición cubre a personas que han sido convertidas al judaismo de acuerdo con procedimientos no ortodoxos, lo que ha motivado fuertes enfrentamientos con las corrientes conservadora y reformista del judaísmo religioso. Se estima que aproximadamente un $25 \%$ de los inmigrantes de la ex Unión Soviética que llegaron a Israel durante la década de 1990 no son judios según la definición ortodoxa.

Por otra parte, uno de los aspectos básicos de la definición de "judio" tiene que ver con la relación con la "Tierra de Israel" (Eretz Israel) y el "Estado de lsrael" (Medina Israel), siendo esta relación uno de los componentes centrales del sionismo. La cuestión de quién es judio no puede estar reducida al tema de la religión ni tampoco al de la "raza" ni etnicidad". Aunque existe una religión común, no es ésta la que une al pueblo judío, sino que por el contrario los judíos

2 Colocamos el término "raza" entre comillas, porque sociológicamente hablando pensamos que es más acertado hablar de "etnicidad" que de "raza" propiamente tal. 
están divididos en diferentes corrientes religiosas. Por su parte, la expresión "raza judía" resulta inapropiada, puesto que no existen categorías raciales compartidas por los judios, que los distingan además de los no judíos (Lange 2000, 25-7).

En este artículo partimos de la hipótesis de que existe una tendencia hacia la conformación de un modelo más descentralizado, laico y fragmentario en la presencia judia en América Latina desde fines del siglo $\mathrm{XX}$, resultado de una profundización de la globalización y radicalización de la modernidad. Este modelo está asociado con cuatro procesos primordiales, que corren en paralelo y están interrelacionados: a) la constitución de identidades judias laicas, con elementos progresistas y liberales, sin que la religión sea necesariamente un elemento central de identificación; b) una tendencia a la consolidación de un espacio religioso ortodoxo, que puede alcanzar a un $10 \%$ de la población; c) la formación de nuevas identidades, muchas de ellas lejos de los marcos comunitarios institucionalizados del judaísmo latinoamericano; d) la consolidación de un debate que incluye tanto al judaísmo institucionalizado como al informal, que se plantea en torno a una crítica al modelo comunitario centralizado. Veremos de qué manera operan estos cuatro procesos en los casos de Argentina y Chile.

\section{IDENTIDADES JUDÍAS LAICAS}

Un elemento sustancial que distingue al judaísmo latinoamericano de otras diásporas de "reciente formación" (como las de Estados Unidos, Canadá, Australia y Sudáfrica), es su diversidad, caracterizada por la incorporación de inmigrantes de casi todos los origenes: "La gran diversidad de colectividades que caracteriza al judaísmo latinoamericano es superada solo por la que existe en Israel" (Avni 1999, 23). Esta diversidad en la presencia judía latinoamericana ha planteado importantes desafios: a) preservación de la identidad judía frente a los procesos de asimilación y emigración; b) necesidad de luchar frente al antisemitismo; c) mantención y consolidación de la relación entre las distintas comunidades entre sí, y entre éstas y el Estado de Israel.

Tomando en consideración algunas tipologias sobre identificación judia en la población judia de Israel y en las comunidades más numerosas de la diáspora, Della Pergola $(1999,478-81)$ observa que el principal elemento de identificación judía no es la religión, sino que está constituido por otros elementos o "modos". El modo de "etnicidad / comunidad"-mantenimiento de redes de asociación judias-tiende a ser el dominante en Israel, Canadá, Gran Bretaña, así como en los tres países latinoamericanos mencionados en el estudio: Argentina, Brasil y México. El de "residuo cultural" - donde la vinculación con el judaísmo es independiente de una conducta personal o compromiso comunitario en la vida colectiva judía- es mayoritario en Estados Unidos, Francia y Rusia. El de "dual judío/no judio"-donde existe un nexo débil o nulo de identificación judía, predominando elementos de contenido no judaico - tiene una importancia más marginal.

Esta tipología es coincidente con estudios específicos sobre población judía y autoidentificación de los judios en los cuatro casos que a continuación se mencionan: Estados Unidos, Argentina (Buenos Aires) y Chile (Santiago). Para un porcentaje importante de la población judia, la religión no es un elemento central de su identidad. En el caso de los judíos estadounidenses, según una encuesta realizada el año 2005 , un $26 \%$ se definió "solo judío", frente a un 
$32 \%$ conservador, $29 \%$ reformista, $10 \%$ ortodoxo y solo $2 \%$ reconstruccionista (The American Jewish Committee 2005). Hay otros dos aspectos o tendencias principales, que emergen a partir de la revisión de encuestas de opinión, con respecto a los judíos estadounidenses. Primero, el campo liberal y progresista está bastante consolidado y está representado por identidades judías en cambio constante, en donde uno de los elementos centrales -más allá del aspecto religioso-es la pertenencia a un grupo nacional y a una cultura determinada. Segundo, la identidad judía está en gran medida relacionada con una actitud y un sentimiento de cercanía hacia Israel y hacia las políticas implementadas por este Estado.

Hay que destacar que las dificultades de la definición de quién es judío -analizado en la introducción- tienen algunas consecuencias para fines de la investigación social empírica. Estos problemas fueron considerados por los demógrafos Schmelz y Della Pergola (1986), quienes desarrollaron un cuadro referencial donde definen tres conceptos diferentes de población judía: a) nuclear, los que se definen como judios en un levantamiento censal; b) extendida, la población nuclear más aquellos que aunque son de origen judío, no se definen como tales; c) ampliada, la población extendida más los miembros no judios de sus familias, tales como cónyuges e hijos en matrimonio mixto. De este modo, el grupo que se define como "judio por religión" forma un núcleo central alrededor del cual existe una población mayor, que incluye a judios por otro tipo de definición, además de familiares no judios (Decol 2001, 148).

En Argentina, un estudio sociodemográfico, conducido por Jmelnizky y Erdei (2005), y organizado por la Asociación Mutual Israelita Argentina (AMIA) y el American Jewish Joint Distribution Committee (AJJD), realizado el año 2005, mostraba algunos temas relacionados con la identidad, posiciones frente a Israel, antisemitismo. Puesto que uno de los principales problemas para llevar a cabo este estudio tuvo que ver con la definición de qué se entendía por "judio", se decidió que se consideraría como tal a toda persona que respondiera a uno de los siguientes cuatro criterios: a) en función de su ascendencia, esto es la persona cuyos padres (ambos o uno de ellos) son judíos o cuyos abuelos (uno o varios) son o hayan sido judios; b) en relación con su autodefinición; c) sobre la base de su definición religiosa; d) por adopción, elección o conversión.

Como componentes principales de la identidad judia, están en primer lugar de importancia el "festejo de celebraciones judias familiares", seguido de "comer comida judía típica" y "familiares contando recuerdos de su pasado judio". También son importantes para la identidad, "escuchar música judia", "escuchar hablar idish/ladino/hebreo" y tener "familiares contando recuerdos del Holocausto". Sin embargo, la participación en grupos judios, la lectura de textos o periódicos judíos, la existencia de amigos judios durante la infancia, son aspectos menos determinantes en la identidad judía.

En cuanto al tema religioso, un $67 \%$ de los judíos bonaerenses se consideró poco o nada observante, frente a un $29 \%$ que se definió bastante o muy observante. Sobre la observancia de Yom Kipur (Dia del Perdón), un $60 \%$ señaló no haber ayunado, en tanto que un $34 \%$ sí lo hizo. Por su parte, un $74 \%$ afirmó no respetar las normas dietéticas establecidas por el Rabinato. Además, la gran mayoria de la población judía argentina no concurre en forma regular ni ocasional a un servicio religioso judío, ni realiza una cena sabática en su hogar. 
Considerando los elementos anteriores, a partir de este estudio demográfico, se concluye que los judios bonaerenses que se autodefinen como tales tienen distintas formas de identificación con el judaísmo, pudiéndose distinguir cinco categorías principales: a) un $30 \%$ son "judios solo por cultura", que se definen como judíos a través de la cultura y lazos familiares; b) un $25 \%$ son "judios por cultura con educación judia, creyentes", los que tienen alguna experiencia formativa judia; c) un $20 \%$ son "judios por cultura con educación judía, no creyentes", los que comparten los rasgos anteriores, con la única diferencia de que no creen en la existencia de Dios; d) un 16\% son "judios por religión y tradición", los que tienen un contenido religioso adscrito a su identidad; e) un $9 \%$ son "judíos por religión y tradición ortodoxa". Esto significa que para el $50 \%$ de la población judía autodefinida del Gran Buenos Aires, la religión no constituye el principal componente de la identidad judía. Para este conjunto, los elementos identitarios centrales son: lazos familiares, memoria histórica (holocausto), educación judía sin creencia en Dios.

En Chile, un estudio sociodemográfico realizado por el Comité Representativo de las Entidades Judias de Chile - CREJ (1995), al igual que el de Argentina y otros similares en Brasil y México, debía resolver qué se entendía por judío. La población del estudio fue definida como hogares que incluyeran al menos a algún miembro que se considerara judío, que hubiera sido criado como judío o cuyos padres fueran de religión judía. De este modo, se incluyeron hogares con miembros definidos como judíos de acuerdo con criterios no religiosos y a hogares integrados por miembros tanto judíos como no judíos. Esta definición de la población del estudio es consistente con los otros estudios llevados a cabo en la Diáspora y, en este caso, corresponderían a una "población judía extendida".

En cuanto a los aspectos más importantes de la identidad judía, se mencionaron "celebrar Pésaj" (68\%), "mantener la tradición judia" (64\%), "trabajar por la justicia social” (63\%), "celebrar las festividades judias" (58\%) y "visitar Israel" (58\%). La observancia de prácticas religiosas -como "respetar las leyes judias" o "participar en servicios en la sinagoga"-adquiere menor importancia en la identidad judía. ¿Cuál es la definición autorreferencial de los judíos chilenos? Un $35 \%$ de los entrevistados se definió como judío conservador; un $23 \%$ como judío no religioso o laico; un 19\% como judio tradicionalista; un 14\% como judio reformista; y un $3 \%$ como judio ortodoxo. Se considera a los judios tradicionalistas como aquellos que se ubican en un nivel intermedio, entre los judios conservadores y los judios reformistas.

Sobre prácticas religiosas, el $15 \%$ contestó participar en servicios religiosos judíos por 10 menos una vez por semana, en tanto que el $82 \%$ respondió ir a la sinagoga en las Altas Fiestas Judias. Pésaj o la pascua judía era la fiesta más respetada, por $85 \%$ de los judíos adultos de la Región Metropolitana. El ayuno de Yom Kipur es cumplido por el $70 \%$ de los judios de Santiago, cifra que contrasta con el $34 \%$ de los judios argentinos que respondió haber ayunado. Sin embargo, un $9 \%$ de las personas respondió que respetaba las normas dietéticas. También se estableció que la proporción de personas que nunca asisten a servicios religiosos o que nunca ayunan en Iom Kipur aumenta de la primera generación a la tercera y cuarta generación en Chile, "lo cual podria indicar el impacto de la mayor distancia generacional de las personas con respecto a sus origenes inmigratorios en la erosión de la identificación judia" (CREJ 1995, 66). 
Esta conformación de identidades judías sin que la religión tenga un lugar central corre paralelo con la constitución de un judaismo laico y humanista, lo que es parte de una tendencia mundial, que tiene su centro en Estados Unidos y que plantea una filosofia centrada en el ser humano, que combina la celebración de la cultura y de la identidad judía con el respeto a los valores humanísticos universales. Este movimiento logra cierta organización en Argentina, al formarse en 1987 la Asociación Argentina por el Judaísmo Laico y Humanista, creando la Revista Judaismo Laico, y considerándose en armonía con el sionismo. La doctrina fundamental es la consideración del laicismo en conformidad con la historia contemporánea del pueblo judío y del sionismo (Feierstein 1999, 220-1).

\section{ESPACIO RELIGIOSO ORTODOXO}

En el caso del mundo judío latinoamericano, junto con la conformación de identidades sin que la religión sea un componente central de identificación, parece consolidarse una tendencia en el sentido de una "vuelta a la religión", que implica la afirmación de un espacio religioso ortodoxo. Del mismo modo que en el islam hay un "retomo a la mezquita" o una "reislamización por abajo" (Etienne 1996, Roy 1996, Kepel 2005), en el caso del judaísmo este retorno a los fundamentos religiosos está sustentado también en los siguientes factores: a) una crisis de las ideologías, especialmente a partir del marxismo; b) la necesidad "del hombre moderno" de volver a valores espirituales, frente a una sociedad consumista y materialista; c) la búsqueda de certezas frente a un mundo de incertidumbres ${ }^{3}$.

Uno de los rasgos fundamentales que afecta a las comunidades judías latinoamericanas consiste en un movimiento de teshuva, que en hebreo significa retomo, que designa un arrepentimiento, y hoy se refiere al proceso a través del cual los judíos, laicos o de otras corrientes religiosas, reevalúan su identidad judia, transformándose en ortodoxos. En esta dirección, se observa, primero a partir de la década de 1970 y luego a partir de 1990, la fundación de nuevos espacios ortodoxos: yeshivot (academias de estudios rabínicos), kolelim (institución de estudios rabínicos para hombres casados) y centros de estudio informales (Topel 2005, 83-4).

Durante la década de 1960, las comunidades latinoamericanas recibieron una influencia importante de las corrientes conservadoras del judaísmo, con la fundación del Seminario Rabínico Latinoamericano, considerado el centro académico, cultural y religioso más importante del movimiento conservador judío. Este seminario, fundado en 1962, que tiene como objetivo principal formar y ordenar a rabinos de toda la región, para difundir y perpetuar la religión judía en las comunidades latinoamericanas, ha graduado a 83 rabinos, incluyendo a seis mujeres, los que ejercen como tales en los distintos países de la región y en Estados Unidos.

3 Entrevistas a los rabinos Roberto Feldmann y Efrain Rozensweig. 2006. Proyecto FONDECYT No 1050053. 
También se han desarrollado formas religiosas más progresistas y liberales, representadas por las corrientes reformista y reconstruccionista del judaismo, así como por el denominado movimiento Renewal, fundado en la década de 1960 en Estados Unidos. Este último se plantea como una corriente mundial y transversal, que ha logrado importancia en Brasil, a través de la Congregacao Israelita Patlista, con sede en Sao Paulo, considerada la agrupación liberal más grande de América Latina. Todas estas corrientes se han caracterizado por mantener una distancia importante con respecto a los sectores ortodoxos, especialmente en cuanto a la concepción del judaísmo, el tema de las conversiones, y tópicos relacionados con la homosexualidad.

Sin embargo, desde la década de 1970 , existe un movimiento mundial de "regreso a la religión" en sus concepciones más ortodoxas. Algunos datos para el caso de Brasil muestran que hasta 1985 existían cinco rabinos ortodoxos en la ciudad de Sao Paulo, cifra que 20 años después sobrepasa los 100, convirtiendo a esta ciudad en una "pequeña Jerusalén". El judaísmo ortodoxo tiene un resurgimiento con la fundación de Beit Chabad en 1970, Binyan Olam en la década del 80, y un rápido crecimiento en la década de 1990 con la fundación de nuevos espacios. En lo últimos 15 años se han construido 15 sinagogas ortodoxas, tres yeshivot, dos kolelim y cinco escuelas. (Topel 2005, 83-93).

En Argentina, donde se estima que alrededor de un $10 \%$ de la población judía pertenece al movimiento ortodoxo, éste está especialmente representado por Jabad Lubavitch, una de las corrientes ortodoxas más importantes a nivel mundial, experimentando un crecimiento constante que va de 480 instituciones en 1975 a alrededor de 2.600 instituciones el año 2000 en todo el mundo. Jabad, fundado en el siglo XVIII en Bielorrusia, corresponde a la sigla de las tres palabras hebreas Jojmá, Biná y Daat, que constituyen la estructura intelectual del hombre: inspiración, entendimiento y conciencia.

Este movimiento se plantea como objetivo central que el pueblo judío cumpla con su misión de seguir los preceptos contenidos en la Torá, para lo cual resulta fundamental combatir el desconocimiento que existe sobre el judaísmo histórico y espiritual, producto de la "emigración y de los traumáticos acontecimientos de este siglo volcánico". Siendo de carácter mundial, Jabad está en todas partes: "En la Rusia del Zar, bajo la dictadura comunista, en la reconstrucción espiritual del mundo judaico post-holocausto, en los frentes de batalla de Israel, en las Universidades del mundo libre occidental, en Nepal y Machu Pichu. En todos lados está Jabad" (Jabad Lubavitch 2005).

Hay que mencionar también al grupo Torá, preocupado del respeto a la ley mosaica, la cual cuenta con la Librería Judaica -que distribuye libros principalmente religiosos-, y con una "Guía para el consumidor Kosher" - con establecimientos en Buenos Aires y en el interior del país. Por su parte, está la Comunidad Adjut Israel, que cuenta con una escuela, con la Yeshiva Jazon Iejezkel creada en 1989, y con la publicación anual del libro Hamadrij Lecashrut, destinado a entregar información sobre la comida kosher. La consolidación de la ortodoxia se expresa también en las elecciones llevadas a cabo en la AMIA, en abril de 2008 , las que fueron ganadas por e] Bloque Unido Religioso, que alcanzó el $38 \%$ de los votos, representando a los grupos ortodoxos y aumentando la polémica con los sectores liberales. 
En lo que se refiere al judaísmo chileno, se percibe también un regreso a los "fundamentos" religiosos, con un crecimiento de las corrientes ortodoxas, lo que tendría tres indicadores centrales: a) el aumento de sinagogas ortodoxas, que antes no existían en Chile, y la mayor participación en las mismas; b) la presencia de rabinos ortodoxos, los que también eran inexistentes; c) el crecimiento del consumo de comida kosher.

Una característica central de este regreso a la ortodoxia es que está constituido por un movimiento de jóvenes. La vuelta a la ortodoxia, en que la "sinagoga se convierte en un referente de lo identitario judio", hay que buscarla hacia fines de la década de 1990, teniendo un hito importante en el accidente de tránsito que tuvieron cuatro jóvenes judíos el año 1998, producto de las "tres V, Vitacura, Vodka y Vitara", esto es un "Chile jaguar, exitoso", en que la comunidad judia "se cambió de Macul a Lo Barnechea". Este fue un hecho fundamental, porque es el momento en que la ortodoxia "despliega una respuesta a una necesidad de la comunidad, que no tenía una narrativa juvenil"4.

Este fenómeno tiene como principales manifestaciones: a) la formación en Santiago, en 1996, de Aish Chile, fundado por un grupo de jóvenes encabezados por el rabino Shoshan Ghoori, siendo la rama chilena el primer centro de habla hispana; b) la formación en 1998 del colegio Maimónides, vinculado a la corriente ortodoxa; c) el crecimiento del consumo y distribución de comida kosher, lo que se reflejaria en la creación de una lista de productos kosher en www. koscherchile.cl, así como en el acuerdo de los rabinos ortodoxos de supervisar plantas de empresas chilenas.

El espacio ortodoxo es muy diverso, con múltiples tendencias y divergencias internas. Algunas diferencias centrales entre los ortodoxos y los ultra ortodoxos, más allá de las definiciones teológicas -lo que escapa al objetivo de este trabajo-, tienen que ver con las distintas posiciones con respecto al Estado de Israel y al sionismo, siendo posible distinguir cuatro grandes subcomientes al interior de este campo (Shafir y Peled 2002, 138-40): a) "acomodación pragmática", que emerge en el movimiento sefaradí establecido en 1902 como una fracción ortodoxa, según la cual los judíos ortodoxos deberían participar en la tarea sionista de construir un Estado judio; b) "acomodación de principio", cuya posición corresponde a un sionismo religioso, que le otorga al proyecto sionista de creación del Estado de Israel una significancia religiosa; c) "rechazo pragmático", que es la posición de los sectores ultraortodoxos, no sionistas, que se caracterizan por su rechazo del sionismo ideológico y político, aunque en términos prácticos lo acepten; d) "rechazo de principio", que ve el sionismo y el Estado de Israel como empresas demoníacas y rechazan cualquier cooperación con él.

4 Entrevista a Jorge Zeballos. 2006. Proyecto FONDECYT 1050053. Zeballos refiere a un accidente ocurrido en agosto de 1998, en Avenida Kennedy, en Santiago, en que murieron cuatros jóvenes judios que iban en un auto a exceso de velocidad, hecho que fue reporteado ampliamente por la prensa nacional y comunitaria. También hace referencia a un proceso en que las distintas organizaciones judias cambian sus sedes desde comunas de clase media como Santiago, Ñuñoa y Macul, hacia aquellas de sectores más acomodados del llamado "barrio alto", como Las Condes, Vitacura y Lo Bamechea. 


\section{FORMACIÓN DE NUEVAS IDENTIDADES}

La constitución de identidades liberales y progresistas, por una parte, y la consolidación de un espacio religioso ortodoxo, por otra, corren paralelo con procesos, modas y corrientes, que se expresan en la conformación de nuevos espacios identitarios judios, los que no corresponden a un judaísmo institucionalizado. Todos estos procesos, algunos de los cuales tienen su origen en las comunidades judías estadounidenses, encuentran cabal expresión en los países latinoamericanos, siendo resultado de la formación y consolidación de un modelo comunitario descentralizado, formado más por identidades en movimiento que por comunidades centralizadas.

En primer lugar, una tendencia hacia la apertura encuentra especial cabida en los jóvenes judíos, a través de la búsqueda de nuevos sentidos de pertenencia, de un nuevo "orgullo judío", de versiones humorísticas del ser judío, que dejen de lado el espacio tradicional que ocupaba la familia o la sinagoga. Ahora, se trata de un estilo de vida, que se expresa en una determinada música, en la lectura de ciertas revistas juveniles y en la realización de reuniones privadas. Esta nueva tendencia es conocida en Estados Unidos como "jewcy" o como "orgullo koolsher", denominación que corresponde, en ambos casos, a un juego de palabras. "Jewcy" ("judío jugoso") reúne las palabras "jew" (judío) con "juicy" (jugoso). "Koolsher" reúne el término inglés "cool" con la palabra hebrea "kosher".

Algunos íconos que alimentan esta "causa identitaria", que tiene su origen en Nueva York, son Woody Allen, reconocido director de cine judio, y la figura de Madonna, en especial por su cambio de nombre a Ester y su adhesión al estudio de la Cábala. En América Latina, esta tendencia se expresa especialmente en los jóvenes judíos que trabajan en la radio, en la televisión y en el teatro, siendo una reacción a la reafirmación religiosa judía que se manifiesta en estas comunidades, logrando fuerte adhesión en los círculos de jóvenes judíos, tanto de Santiago como de Buenos Aires. En este sentido, el ser judio y la creencia en Dios se visualizan como dos cosas distintas y que queda expresado en la siguiente frase: "Creer en Dios es una opción que está desligada de ser judío" (Wassermann 2005, 28).

Relacionados con estos nuevos espacios de apertura, están la constitución de grupos, modas y tendencias en Argentina, como lo ejemplifican Yok y Grupo Otra Mirada. Lo primero es una propuesta social y cultural de un "judaismo integrador, diverso y dinámico", que plantea vivir el "judaísmo a tu manera", a través de manifestaciones intelectuales y artisticas, que incluyen cine, música, cocina, deportes, pensamiento, recreación y moda. Este proyecto es resultado de los datos mostrados anteriormente, que señalan que para parte importante de la población judía de Buenos Aires, la religión no es el componente principal de la identidad judía, sino que ella está conformada por elementos como la cultura, la música, la literatura. De este modo, "Yok nace con la finalidad de contribuir al desarrollo de una comunidad pluralista", dando nuevo significado a los valores judíos y explorando formas de relación en marcos informales (Yok 2006).

Grupo Otra Mirada, por su parte, está constituido por un grupo de jỏvenes, que se reúnen en julio de 2006, en momentos de la guerra entre Israel y Hezbolá, con el objetivo de generar "un espacio de debate" acerca de este conflicto en el marco de la Facultad de Filosofia y Letras de la Universidad de Buenos Aires. Ha participado en una serie de actividades relacionadas con la 
lucha contra el antisemitismo, conmemoración de los atentados contra la AMIA y la Embajada de Israel, diálogos de paz. En un Blog colocado en noviembre de 2007 se señala que el grupo fue firmante de una carta oponiéndose a la decisión de la AMIA de no permitir en sus cementerios el entierro de judíos conversos por los movimientos reformista y conservador.

En Chile, está la creación de versiones humorísticas del ser judío, como Hagolem Chiliani, revista de humor que toma como base el judaísmo, utilizando la sátira y la ironía a través de títulos como los siguientes: a) "el travestismo étnico: la nueva moda kehilati"; b) "Haskamá rabínica da legitimidad para Hagolem"; c) "Nico, el flaite de Lo Hermida habla de sus vecinos judios"; d) "¿Se ha preguntado cómo sería si Navidad fuese un día de fiesta judía?". En una de sus páginas se lee como advertencia: "Hagolem Chiliani es una parodia pensada con fines meramente chocarreros Si ofende a cualquier persona, nos disculpamos por adelantado para no llevar tanto peso en el próximo Tashlij"'.

En segundo lugar, como parte de este proceso de apertura que incluye la vida judia latinoamericana, estamos en presencia de la conformación de identidades que -junto con rescatar el elemento judaico, definido en términos amplios y más allá de la religión-, rescatan una identidad sustentada en la orientación sexual y en la sexualidad. De este modo, se forma en Argentina la organización Keshet, que se plantea el desafio de lograr que la comunidad judía incluya plenamente a gays, lesbianas, bisexuales y transgéneros en su seno, bajo el principio general de que todos los judios "tienen el derecho de vivir su judaísmo plenamente". También hay que mencionar a los Judios Argentinos Gay (JAG), que nacen como una respuesta a la poca importancia que las organizaciones judias tradicionales dan a este tema. No obstante, JAG participa en la Juventud de la AMIA y, una vez obtenida la personalidad juridica, busca integrarse como organización reconocida a la DAIA. El presidente de JAG, Diego Guinecin, hace ver que durante el holocausto también fueron perseguidos los homosexuales y que existieron homosexuales judíos que debían llevar una estrella de David de color rosado. Resulta, por lo tanto, una ironía la discriminación de éstos por parte de la comunidad judía argentina.

En Chile, está la constitución de un grupo judío gay, en noviembre de 2005 , denominado Grupo Janucá, nombre que adoptó por haberse realizado una primera reunión con motivo de esta fiesta judía. Su fundación se dio en el marco de exhibición del documental Trembling before God, que narra vivencias de judios ortodoxos que han asumido en forma abierta su orientación homosexual. Existen instancias que muestran una apertura de determinados sectores judios, especialmente de los jóvenes, hacia el tema gay. Algunos pasos institucionalizados se han dado a partir de los contactos de la Juventud Judía de Chile con el Movimiento de Liberación e lntegración Homosexual (MOVILH) en el marco de actos reivindicativos de las minorias en el pais y en organizaciones conjuntas opuestas a la presencia de movimientos neonazis (Wassermann 2007, 6-7). En este sentido, en algunas oportunidades ha sido más fácil para la Juventud Judia acercarse a dirigentes homosexuales que a dirigentes palestinos. Estos contactos se han facilitado porque los movimientos homosexuales consideran a la sociedad israelí como "tolerante y no 
fundamentalista religiosa", lo que queda ejemplificado con la realización anual del "Gay Parade" (marcha del orgullo gay) en Tel-Avivs.

Estas nuevas identidades corresponden a un fenómeno social que se diferencia del judaísmo tradicional e institucionalizado, que se manifiesta en la creación de talleres de reflexión, encuentro de artistas y profesionales, versiones humoristas del ser y del quehacer judio. Se trata de identidades diversas donde impera el disenso, con múltiples opciones para ser judio, en donde la cultura judia aparece atravesada también por la fragmentación moderna (Herszkowich 2006, 109). Además, debemos considerar la importancia que tiene internet en la formación de estas identidades, al permitir un nexo más fluido, así como la existencia de foros de discusión, listas de correo, libros de visitas, los que operan como redes sociales desterritorializadas, convirtiéndose en una alternativa para múltiples grupos, personas y causas, uniendo a los judios y no judíos de todas las edades, que buscan conocer sobre judaísmo, obtener noticias de Israel y del conflicto árabe-israelí y/o debatir sobre cualquier tema, sea real o imaginario, en forma seria o humorística.

\section{DISIDENCIAS Y FRAGMENTACIONES EN LAS COMUNIDADES JUDIAS}

Los procesos anteriores están relacionados con una tendencia a la conformación de comunidades judías más fragmentadas y descentralizadas, repartidas en múltiples organizaciones de carácter social, deportivo, cultural, religioso. En esta dirección se consolida una crítica a la centralización comunitaria, la que observaremos a partir de dos hechos emblemáticos que involucran cuestionamientos recientes a los organismos centrales del judaísmo argentino y chileno, respectivamente ${ }^{6}$.

Las críticas a la AMIA se remontan a los atentados realizados en Buenos Aires en 1992 contra la Embajada de Israel y en 1994 contra el edificio compartido por esta organización y la DAIA. La creación de Memoria Activa, organización independiente formada por los familiares del atentado de 1994, con el objetivo de esclarecer este hecho, es resultado de estas divergencias. Durante 1998 y 1999, se desató una polémica entre la AMIA y la DAIA al descubrirse la desaparición de parte importante de los 12 millones de pesos argentinos que el gobiemo argentino había entregado como subsidio especial a la comunidad judía para paliar las consecuencias del atentado de 1994 (Feierstein 1999, 434-5). Más recientemente, un debate que ha tenido lugar al interior de la comunidad judía argentina es el que dice relación con los entierros de los conversos en cementerios judíos y que tiene como punto de partida la decisión de la AMIA de seguir las directrices religiosas del Gran Rabinato (ortodoxo) de Israel.

Entrevista a Jorge Zeballos. 2006. Proyecto FONDECYT 1050053.

Hubo también cuestionamientos importantes a las organizaciones centrales del judaísmo argentino (DAIA, AMIA) y chileno (CREJ) por su papel en los regímenes militares de Argentina y Chile, respectivamente, tema que escapa a los objetivos de este articulo. 
En agosto de 2007, la Mutual judía emitió una declaración pública, en la cual se enfatiza que el cementerio administrado por esta organización es de carácter ortodoxo y religioso, está regido por la Halajá, y obedece a los "principios del Superior Rabinato de Israel". Según estas normas, solo se permite "enterrar en nuestros cementerios a judios, hijos de padre y madre judíos, o solo de madre judía". Esto plantea un problema en el caso de fallecer una persona "convertida por los movimientos conservador y reformista cuya conversión no es reconocida por el Superior Rabinato de Israel". En esta misma declaración, se agrega que "no podrán ser recibidos, ni aceptados dichos conversos en vida, como socios de la Kehilä". Tampoco se podrá interpretar que la AMIA legitima los casamientos mixtos, los "que amenazan permanentemente la existencia de nuestro pueblo en la diáspora" (AMIA 2007).

Esta resolución implicó una reacción inmediata del movimiento conservador argentino, el cual expresó "su consternación y rechazo vehemente" a la misma, su preocupación por la negativa a que los conversos por estos movimientos puedan ser en vida socios de la Mutual, así como por "la prohibición de que mujeres Rabinas puedan oficiar sepelios y otras ceremonias en los mismos cementerios". La declaración del movimiento considera que éstas son "medidas discriminatorias jamás enunciadas ni practicadas en nuestra querida kehila desde su fundación" (Movimiento Conservador 2007).

La prensa comunitaria ha seguido de cerca este debate, dando cobertura a ambas posturas, las que aparecen como irreconciliables, mostrando una vez más la diversidad de tendencias al interior del judaismo religioso y la brecha que separa a las corrientes reformistas, conservadoras y liberales, por una parte, de las ortodoxas, por otra:

"Es necesario para la comunidad argentina rever este tema. La ortodoxia no puede querer seguir teniendo el mandato absoluto y debe reconocer a los Rabinos conservadores y reformistas. Los conversos abrazaron el judaísmo por propia decisión, la gran mayoría por amor a las fuentes (...). Todos somos judíos, desde el más laico hasta el más ultraortodoxo. La AMIA se equivocó con la decisión" (Dubesarsky 2007).

"Estoy totalmente de acuerdo con respetar el Shuljan Aruj, las conversiones hechas por el Seminario Rabínico lamentablemente no son válidas, y por ello, esa gente sencillamente no es Judía y por ende no pueden ser enterradas en el cementerio Judío. Ojalá puedan algún día en el Seminario, hacer todo como dice la Halajá, entonces quizás allí, por fin podremos tener real unidad los Judios" (Linietzky 2007).

De este modo, se produce un debate y una fuerte critica a la "centralización comunitaria" que todavia representarían las organizaciones judías, se trata de una oposición entre ciudadanía libre y un "ghetto virtual": "Salir del ghetto de la Comunidad Institucionalizada es como volver a salir de Egipto", señala el rabino Sergio Bergman, demandando una ciudadanía activa que rompa los lazos con las "instituciones emblemáticas de la comunidad judia", las que no son centrales ni representativas, sino que responden a la organización del Estado argentino.

En Chile, en algunos sectores judios, especialmente en los más progresistas -esto es el movimiento Hashomer Hatzair, Judios por Lagos, Centro Progresista Judio-, ha existido cierta desafección y desvinculación con respecto a lo que son los órganos institucionales y centrales de 
la comunidad judía. Esta "disidencia" se remonta al periodo de la dictadura militar y a un debate -que escapa a los objetivos de este estudio- sobre el papel que tuvo la dirigencia comunitaria durante ese régimen. Hacia fines de 1980, se formó un grupo denominado "Judíos por el No", los que conformaron un espacio progresista, opuesto al gobierno militar de Augusto Pinochet, que posteriormente van a constituir el grupo "Judios por Lagos", como un espacio de apoyo al gobierno del presidente Ricardo Lagos y ajeno "a las comunidades judias institucionalizadas". En el ámbito comunitario, esta agrupación realizó un "acto de protesta por la utilización del Estadio Israelita Maccabi a mediados de los ochenta en apoyo de actividades de la represión". Paralelamente, esta "disidencia comunitaria" se manifestará a partir de los hechos vinculados con el conflicto israelí-palestino. Cuando se produce la Segunda Intifada, ésta tiene consecuencias importantes para la comunidad judia: por una parte, "la unió en el sentido de que había un entorno hostil a lo sionista"; por otra, "terminó por alienar o separar a los elementos, individuos o grupos de la comunidad judia que ya eran críticos a la institucionalidad, aquellos más de izquierda o secular"?

En lo que respecta a Hashomer Hatzair, ha existido una tendencia de este movimiento a asumir una posición crítica frente al enfoque oficial mantenido por la Comunidad Judía de Chile y la Federación Sionista de Chile en relación con los problemas de Israel, el Medio Oriente, el quehacer comunitario y la concepción de lo que significa ser judío y ser sionista. La relación del movimiento con los órganos comunitarios centrales del judaísmo chileno no ha sido fácil ni fluida. Un importante debate se dio en el mes de mayo de 2006: con motivo de Yom Hazikaron (Día del Recuerdo), primero, y de Yom Haatzmaut (Día de la Independencia), después, Hashomer Hatzair preparó un discurso en el cual se cuestionaba al gobiemo israelf y, por lo tanto, la posición oficial de la dirigencia comunitaria chilena en el sentido de no cuestionar a ningún gobierno de Israel: "ser sionista no significa apoyar incondicionalmente a un gobierno porque no podemos confundir gobierno con Estado" (Hashomer Hatzair 2006, 4).

Sin embargo, este discurso no se pronunció, lo que, según dirigentes de Hashomer, se debió a que fue censurado y "omitido de la ceremonia". En una carta dirigida a La Palabra Israelita, el dirigente de Hashomer Hatzair, Claudio Mandler, acusó a la Comunidad Judia de hacer una marginación deliberada, "solo por ser un foco crítico, ideológico, profundo, el cual molesta y hace ruido a la hegemonia del liderazgo comunitario", la que cree tener una "receta única de lo que significa ser judio, o ser sionista, o ser parte de la comunidad". Se agrega que esta política de exclusión promueve la asimilación de muchos jóvenes por pensar diferente, lo que es propio de regímenes militares, pero "no de una comunidad judía en la diáspora, que debiera acoger y apoyar, en vez de censurar y excluir". Al mismo tiempo, se señala que hay diferentes formas de ser judio:

"Ser judío no es un monopolio. No hay una receta para ser sionista (...) Somos tan judíos como el resto y tan sionistas como todos (...). Aunque no les guste a todos, seguiremos siendo críticos de las injusticias en la comunidad, en Chile, en el Medio Oriente y en el mundo. 
Espero que en el futuro este tipo de comportamientos no ocurran, ya que los únicos perdedores serán la Comunidad Judía de Chile y el Estado de Israel" (Mandler 2006a, 12).

La respuesta a esta acusación vino del entonces presidente de la Federación Sionista de Chile, Roberto Muñoz, quien solicitó a La Palabra Israelita la publicación del discurso en cuestión, al tiempo que señaló no considerar que Hashomer Hatzair sea "un grupo marginado de la Comunidad Judia". No obstante esta apreciación, Muñoz destacó que los planteamientos del movimiento contienen "una serie de apreciaciones que no se compadecen con el significado que tiene el Estado de Israel para la Comunidad Judeo Sionista de Chile". Destacando el "apoyo incondicional" al gobiemo de Israel, el dirigente comunitario alentó a irse a Israel a quienes sean críticos del gobiemo israeli:

"Como sionista, apoyo en forma incondicional al gobierno de Israel, de izquierda, centro o derecha, toda vez que éste sea elegido democráticamente por el pueblo de Israel. Por último, cualquier judío que sienta la necesidad de 'cuestionar el actuar del gobierno israeli', solo tiene que hacer sus valijas, tomar el avión, y participar activamente dentro del proceso democrático que impera en Israel" (Muñoz 2006, 4).

En suma, la pérdida de confianza en las instituciones comunitarias, las fuertes críticas a las organizaciones centralizadas, los avances tardios en la causa por el atentado contra la AMIA en el caso argentino, los problemas económicos y financieros de estas comunidades, los efectos de la globalización cultural, muestran la consolidación de una tendencia hacia un modelo judío descentralizado y fragmentado, en donde las nuevas identidades jugarán un rol cada vez más importante al interior de las comunidades judías. Probablemente éste sea un paso necesario para el enriquecimiento y florecimiento del mundo judío argentino y chileno, y para que éste mantenga un rol de primer orden en la consolidación de nexos con otras comunidades, árabes y musulmanas, fortaleciendo un diálogo intercultural e intercivilizacional con ellas a partir del reconocimiento de la diversidad de corrientes, tendencias y procesos.

\section{CONCLUSIONES}

El mundo judío constituye una amplia diversidad de procesos, tendencias y modelos cambiantes y contradictorios entre sí, que parte por algunas polémicas que están en el origen mismo del problema de ¿quién es judio? y de cuál es la relación con el sionismo y con el Estado de Israel. En el judaísmo latinoamericano, con una diversidad mayor a la de otras comunidades judías de "reciente formación", hay en desarrollo un modelo más descentralizado y fragmentado, que se caracteriza por una tendencia que parece marcar la presencia judía en la región desde fines del siglo XX, y que está asociado con cuatro procesos primordiales, que corren en paralelo y que están interrelacionados.

En primer lugar, está la formación de identidades y organizaciones judías, con elementos progresistas y liberales, donde la religión no es necesariamente un aspecto central de identificación, sino que son otras las cuestiones que definen la pertenencia judia, como la tradición, la memoria histórica, el holocausto, la educación, el sentimiento de cercanía con Israel, el sentido de pertenencia a un grupo nacional. Se trata esencialmente de un movimiento humanista y laico, cuya 
doctrina fundamental es la consideración del laicismo en armonia con la historia contemporánea del pueblo judío y del sionismo.

En segundo lugar, hay una tendencia a la consolidación de un espacio religioso ortodoxo, que puede alcanzar a un $10 \%$ de la población judía, que tiene algunas de sus principales manifestaciones en el aumento de sinagogas, centros de estudio y escuelas ortodoxas; crecimiento en el consumo de comida kosher y creación de listas de estos productos, supervisados por rabinos ortodoxos; proceso de "regreso" a las tradiciones ortodoxas por parte de jóvenes judíos, cuyos padres y familiares han asumido concepciones más liberales del judaísmo.

En tercer lugar, existe la formación de nuevas identidades, las que no tienen cabida en las organizaciones centrales, a partir de la constitución de tendencias, modas o procesos, que reivindican un lugar a los judios gay, un "judaísmo a tu manera", un "orgullo de ser judío", los que no constituyen un judaísmo institucionalizado y se dan en un marco lejano al espacio tradicional que ocupaba la familia, la sinagoga o la escuela. Como parte de este proceso, estamos en presencia de la conformación de identidades que -junto con rescatar el elemento judaico, definido en términos amplios y más allá de la religión-, reivindican nuevos sentidos de pertenencia. Estas nuevas identidades, que son respuesta a los procesos de globalización, corresponden a un fenómeno social que se diferencia del judaísmo tradicional, y que incluye sentidos diversos de identificación, donde impera el disenso, con múltiples opciones para ser judío.

En cuarto lugar, se consolidan debates al interior de las comunidades judias, los que dan cuenta de la fragmentación que ocurre en la vida judía, como parte de los procesos de globalización que definen la vida contemporánea. En Argentina, a las discordancias por el caso relacionado con la causa AMIA, se une un debate que dice relación con los entierros de los conversos en cementerios judios, lo que ha llevado a criticar la centralización comunitaria. En Chile, diferentes sectores del mundo judío cuestionan la posición oficial de la dirigencia comunitaria chilena en el sentido de mantenerse incondicionales a todos los gobiernos de Israel.

Desde fines del siglo XX parece reforzarse un modelo judío laico, progresista, descentralizado, que es resultado de los procesos de globalización y crisis identitarias que se viven en un periodo de radicalización de la modernidad. Al mismo tiempo y, como efecto de estos mismos procesos, este modelo laico podrá verse enfrentado a un crecimiento de posiciones ortodoxas judias, tanto en Israel como en la diáspora, que lleven a una polarización y choque con patrones identitarios más exclusivos. En un polo, estarian formas más inclusivas, de mayor apertura y diálogo, en donde el judaísmo y lo judio se definan en un sentido cada vez más plural. En otro polo, encontraríamos formas más excluyentes, que abogarán porque la religión y las concepciones más ortodoxas de la misma prevalezcan en la definición del ser y del quehacer judío.

\section{BIBLIOGRAFÍA}

\section{AMIA}

Texto emitido por la AMIA sobre entierros en cementerios judíos. Itón Gadol. [En línea] http:// www.itongadol.com.ar/shop/detallenot.asp?notid=18401 [consulta: 08 agosto 2007]. 
AVNI, Haim

Presentación de las comunidades judías en América Latina

En Encuentro y alteridad. Vida y cultura judia en América Latina

México, Fondo de Cultura Económica, Universidad Nacional Autónoma de México

Jerusalén, Universidad Hebrea de Jerusalén, 1999, pp. 15-31.

\section{BERGMAN, Sergio}

Ciudadanía activa Itón Gadol. [En línea]

http://www.itongadol.com.ar/shop/detallenot.asp?notid=16014 [consulta: 14 octubre 2006].

\section{BLOG de Grupo Otra Mirada}

[En linea] http://omirada.blogspot.com/2007/09/grupo-otra-mirada-cumple-1-ao.html [comentario colocado noviembre 8,2007$]$.

\section{COMITÉ REPRESENTATIVO DE LAS ENTIDADES JUDÍAS DE CHILE (CREJ)}

Estudio sociodemográfico de la comunidad judia de la Región Metropolitana.

Santiago, American Joint Distribution Committee, 1995.

DECOL, René Daniel

Judeus no Brasil: explorando os dados censitarios

Revista Brasileira de Ciencias Sociais 46: 147-60, 2001.

\section{DELLA PERGOLA, Sergio}

Encuentro y alteridad. Vida y cultura judia en América Latina

Ed. Judit Bokser Liwerant y Alicia Gojman Backal

México, Fondo de Cultura Económica, Universidad Nacional Autónoma de México

Jerusalén, Universidad Hebrea de Jerusalén, 1999.

DUBESARSKY, Alejandro

Comentarios Itón Gadol. [En línea]

http://www.itongadol.com.ar/shop/detallenot.asp?notid=18532 [consulta: 09 agosto 2007].

ETIENNE, Bruno

El islamismo radical

Madrid, Siglo XXI, 1996.

FEIERSTEIN, Ricardo

Historia de los judios argentinos

Buenos Aires, Ameghino, 1999.

HASHOMER Hatzair

Discurso de Iom Haatzmaut

La Palabra Israelita, mayo 19, 2006. 
HERSZKOWICH, Enrique

Historia de la comunidad judia argentina

Buenos Aires, DAIA, CES., 2006.

MOVIMIENTO Conservador

Comunicado del movimiento masortí (conservador) en la República Argentina

Itón Gadol, agosto 5. [En línea] http://www.itongadol.com.ar/shop/detallenot.asp?notid=18382 [consulta: 08 agosto 8,2007 ].

JABAD Lubavitch

¿Quiénes somos? http://www.jainet.org/quienesomos.htm [consulta:06 octubre 2005].

JMELNIZKY, Adrián, y Ezequiel ERDEI

La población judia de Buenos Aires. Estudio sociodemográfico

Buenos Aires, AMIA, Joint, 2005.

KEPEL, Gilles

La Revancha de Dios

Barcelona, Editorial Alianza, 2005.

LANGE, Nicholas de

El judaismo

Madrid, Cambridge, 2000.

LINIETZKY, Norberto

Comentarios. Itón Gadol. [En línea]

http://www.itongadol.com.ar/shop/detallenot.asp?notid=18545 [consulta09 agosto 2007].

LA PALABRA ISRAELITA

Yossi Beilin pide reconocer a judios por parte paterna

Diciembre 22, 2006.

MANDLER, Claudio

Carta del dirigente de Hashomer Hatzair

La Palabra Israelita, mayo 12, 2006a.

MANDLER, Claudio

Respuesta de Hashomer Hatzair a Roberto Muñoz.

La Palabra Israelita, junio 2, $2006 \mathrm{~b}$.

MUÑOZ, Roberto

Federación Sionista responde

La Palabra Israelita

mayo 19, 2006. 
Organización Sionista Mundial. 2006. Ley del Retorno. Texto completo. [En línea] http://www.masuah.org/ley_del_retorno.htm [consulta 12 septiembre 2006].

ROY, Olivier

Le néofundamentalism islamique ou l'imaginaire de l'oummah

Esprit 4: 80-107.1996.

SCHMELZ, U.O., y Sergio DELLA PERGOLA

La demografia de los judios de Latinoamérica

Rumbos 15: 17-38.1986.

SHAFIR, Gershon, y Yoav PELED

Being Israeli. The Dynamics of Multiple Citizenship

Cambridge, Cambridge University Press. 2002.

SORJ, Bernardo y Nilton BONDER

Judaísmo para el Siglo XXI

Buenos Aires, Lilmod, 2006.

THE AMERICAN JEWISH COMMITTEE

Annual Survey of American Jewish Opinion. [En línea]

http://www.ajc.org/site/apps/nl/content3.asp?c=ijITI2PHKoG\&b=846741\&ct=1740283

[consulta 05 enero 2006].

TOPEL, Marta F.

O movimento de Teshuvá em Sao Paulo e o esgotamento do judaísmo secular no Brasil: algunas reflexoes

Judaica Latinoamericana 83-93, 2005.

WASSERMANN, Isaías

Entrevista al actor Bastián Bodenhofer

La Palabra Israelita

Septiembre 30, 2005.

WASSERMANN, Isaías

Se abren las puertas para los judíos gay

La Palabra Israelita

Agosto 10, 2007

Yok. Acerca de Yok

[En línea] http://www.yoktime.com/acerca.aspx [consulta: 15 octubre 2006]. 